\title{
INFLUENCE OF ONLINE IDENTIFICATION METHODS ON THE NONLINEAR Process CONTROL
}

\author{
Lubomír Macků \& David Novosad
}
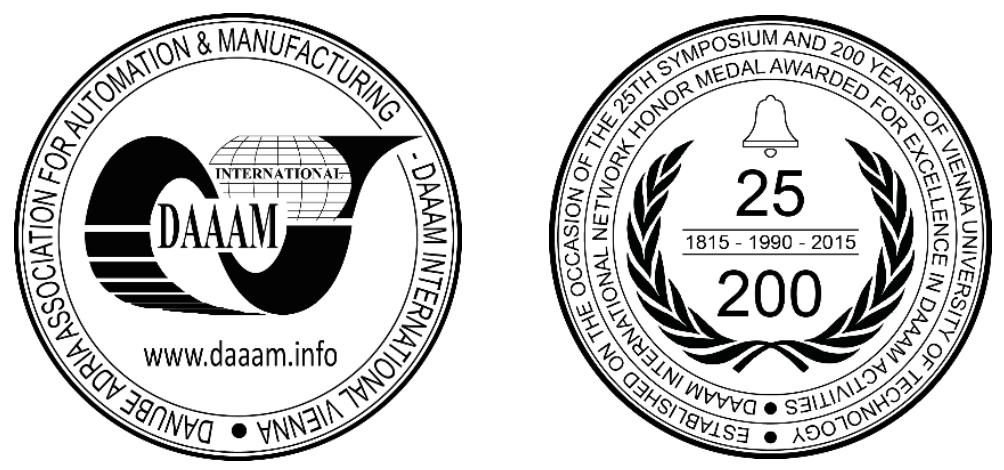

This Publication has to be referred as: Macku, L[ubomir] \& Novosad, D[avid] (2017). Influence of Online Identification Methods on the Nonlinear Process Control, Proceedings of the 28th DAAAM International Symposium, pp.0216-0223, B. Katalinic (Ed.), Published by DAAAM International, ISBN 978-3-902734-11-2, ISSN 1726-9679, Vienna, Austria

DOI: $10.2507 / 28$ th.daaam.proceedings.029

\begin{abstract}
Control of systems with strong nonlinearities is impossible or difficult by common linear systems methods. Sometimes the system linearization around the working point can be a solution, but it is not always applicable. Other, better possibility, is using online system identification during the whole process and based on it the controller parameters changing. In this case, the control is closely dependent on the process identification quality. In this paper, the quality of the control process using different identification methods and their settings is observed. A nonlinear process model based on the exothermic semi-batch reactor is used and the whole process is simulated using data of real process. Results are discussed.
\end{abstract}

Keywords: nonlinear system; online identification; exponential forgetting; semi-batch reactor; process control

\section{Introduction}

Linear systems control is usually done by means of controllers which parameters are set to fixed values. For the PID controller, for example, individual components P, I and D are constant. It is possible here because the linear system can be described by a linear function, and therefore also the linear controller satisfies the entire range of possible input variables.

For nonlinear systems such settings are not possible. Due to the nonlinearities, for example, the controller settings at the beginning of the process may differ significantly from the settings around the permanent operating point. Sometimes it may be possible to divide the control process into smaller individual parts with different local working points and use them as the sub-controller settings. However, in the event of a controlled system deviation from the expected behaviour, a complete failure of control may occur. Sometimes robust or adaptive controllers can be used to overcome the possible problems. These controllers are able to work even with slight system parameters changing, as shown for example on a continuous stirred tank reactor by Zavacka in [1] and by Vojtesek in [2] or on a drying process by Plsek and Matusu [3]. Also the application of model based control can be very powerful for such nonlinear systems as a solid-state fermentation which was introduced by Bück [4]. Again, if the model or its parameters do not match the actual process, there is a high risk of failure. 
Another, more resilient solution is the continuous adjustment of the controller based on the current behaviour of the controlled system. It concerns the controlled system parameters real time identification, so-called online identification. Thanks to online identification, it is possible to adjust the controller settings to the current state of the system.

Any system deviations from the expected behaviour then have no effect on the control itself. The above mentioned procedure is also utilized in this article. Namely, a control process running in an exothermic semi batch chemical reactor is described here. This process is also strongly nonlinear.

The temperature profile in batch and semi-batch exothermic reactors usually follows three stages [5]: (i) heating of the reaction mixture up to the desired reaction temperature, (ii) maintenance of the system at this temperature and (iii) cooling in order to minimize the by-products formation. Any controller used to control such system must be able to take into account these different stages.

In the literature some papers have been published which discuss the control of a batch or semi-batch reactor. A global linearization control strategy with online state and parameter estimation was applied for a polymerization reactor in [6]. However, the authors concluded that the implementation of the proposed method is still difficult due to the missing support of required mathematical functions. The other approach was used in the study [7], where the authors applied a dual-mode (DM) control improved by iterative learning technique. Simulations showed that the proposed method can enhance the conventional DM control with modest effort. For rapid and suitable reference-trajectory tracking a selfadaptive predictive functional control algorithm by Škrjanc was recommended [8]. This approach was successful in a reactor with switching between cold and hot water in the inlet. Neural network was applied to similar system [9] to accommodate the online identification of a nonlinear system. The authors found this strategy effective in identification and control of a class of time-varying-delayed nonlinear dynamic systems.

This paper presents results of experiments obtained by simulations of the semi batch reactor control. For the control the Ziegler-Nichols controller with filtration of D-component (ZNFD) using online identification was used. The paper is organised as follows: In section 2, the semi-batch reactor and the ZNFD controller with online identification methods are described, section 3 presents simulation results and section 4 concludes the current work and suggests new areas for investigation.

\section{Methods section}

\subsection{Semi-batch reactor model}

A mathematical model of the semi batch chemical reactor used for simulations is defined here. Its scheme is shown in Fig 1.

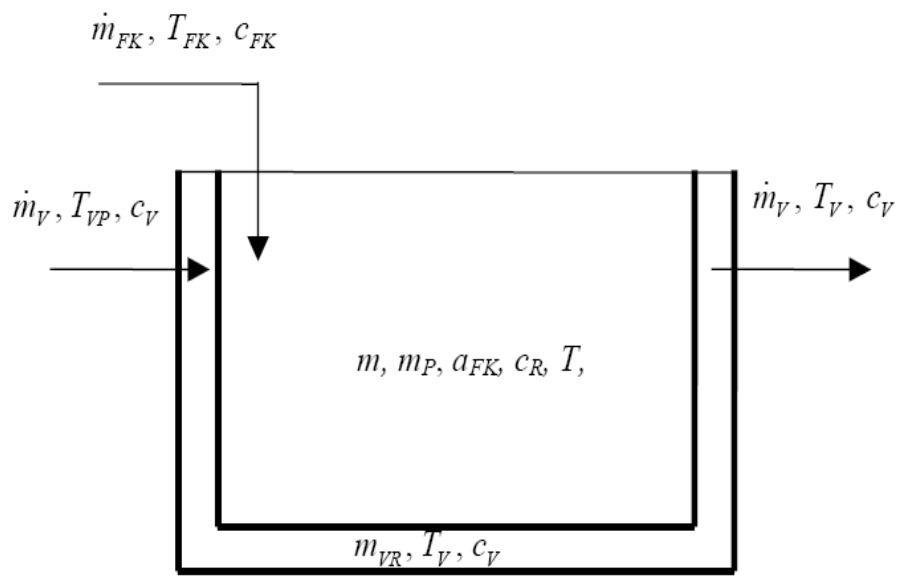

Fig. 1. Chemical reactor scheme

The differential equations 1-4 corresponding to the chemical reactor mathematical model are:

$$
\begin{aligned}
& \dot{m}_{F K}=\frac{d}{d t} m(t) \\
& \dot{m}_{F K}=k m(t) a_{F K}(t)+\frac{d}{d t}\left[m(t) a_{F K}(t)\right] \\
& \dot{m}_{F K} c_{F K} T_{F K}+\Delta H_{r} k m(t) a_{F K}(t)=K S\left[T(t)-T_{V}(t)\right]+\frac{d}{d t}\left[m(t) c_{R} T(t)\right]
\end{aligned}
$$




$$
\dot{m}_{V} c_{V} T_{V P}+K S\left[T(t)-T_{V}(t)\right]=\dot{m}_{V} c_{V} T_{V}(t)+m_{V R} c_{V} T_{V}^{\prime}(t)
$$

The reactor model comprises the total mass balance (1), reaction component mass balance (2), the enthalpy balance (3) and coolant heat balance (4). Further variables and the parameters of the reactor model are listed in Table 1. In (2) $k\left[\mathrm{~s}^{-1}\right]$ is the reaction rate constant expressed by the Arrhenius equation (5):

$$
k=A e^{-\frac{E}{R T(t)}}
$$

\begin{tabular}{|c|c|c|}
\hline \multirow{2}{*}{ (1) } & $\dot{m}_{F K}\left[\mathrm{~kg} . \mathrm{s}^{-1}\right]$ & Mass flow of the entering reaction component \\
\hline & $m(t)\left[\mathrm{kg} \cdot \mathrm{s}^{-1}\right]$ & Accumulation of the in-reactor content \\
\hline \multirow{3}{*}{ (2) } & $a_{F K}(t)[-]$ & Mass concentration of the reaction component \\
\hline & $m(t)[\mathrm{kg}]$ & Weight of all reaction components in the system \\
\hline & $k\left[\mathrm{~s}^{-1}\right]$ & The reaction rate constant \\
\hline \multirow{8}{*}{ (3) } & $c_{F K}\left[\mathrm{~J} \cdot \mathrm{kg}^{-1} \cdot \mathrm{K}^{-1}\right]$ & reaction component specific heat capacity \\
\hline & $c_{R}\left[\mathrm{~J}^{\mathrm{kg}} \mathrm{kg}^{-1} \cdot \mathrm{K}^{-1}\right]$ & Reactor content specific heat capacity \\
\hline & $T_{F K}[\mathrm{~K}]$ & reaction component temperature \\
\hline & $\Delta H_{r}\left[\mathrm{~J}_{\mathrm{kg}}^{-1}\right]$ & Reaction heat \\
\hline & $K\left[\mathrm{~J} \cdot \mathrm{m}^{-2} \cdot \mathrm{K}^{-1} \cdot \mathrm{s}^{-1}\right]$ & Conduction coefficient \\
\hline & $S\left[\mathrm{~m}^{2}\right]$ & Heat transfer surface \\
\hline & $T(t)[\mathrm{K}]$ & Temperature of reaction components in the reactor \\
\hline & $T_{v}(t)[\mathrm{K}]$ & Temperature of coolant in the reactor double wall \\
\hline \multirow{4}{*}{ (4) } & $\dot{m}_{v}\left[\mathrm{~kg} \cdot \mathrm{s}^{-1}\right]$ & Coolant mass flow \\
\hline & $c_{v}\left[\mathrm{~J}^{\mathrm{kg}}{ }^{-1} \cdot \mathrm{K}^{-1}\right]$ & Coolant specific heat capacity \\
\hline & $T_{v p}[\mathrm{~K}]$ & Input coolant temperature \\
\hline & $m_{v R}[\mathrm{~kg}]$ & Coolant mass weight in the reactor double wall \\
\hline \multirow{3}{*}{ (5) } & $A\left[\mathrm{~s}^{-1}\right]$ & Pre-exponential factor \\
\hline & $E\left[\mathrm{~J}^{\mathrm{mol}}{ }^{-1}\right]$ & Activation energy \\
\hline & $R\left[\mathrm{~J} \cdot \mathrm{mol}^{-1} \cdot \mathrm{K}^{-1}\right]$ & Gas constant \\
\hline
\end{tabular}

Table 1. Reactor model variables and parameters

\subsection{Ziegler-Nichols controller with filtration of D-component using Tustin approximation (ZNFD)}

As was already mentioned, to reach desired control properties in minimum time, ZNFD was used to calculate the temperature trajectory. Control law (6):

$$
u_{k}=q_{0} e_{k}+q_{1} e_{k-1}+q_{2} e_{k-2}-p_{1} u_{k-1}-p_{2} u_{k-2}
$$

where $e_{k}$ is control error $\left(e_{k}=w_{k}-y_{k}\right)$ and controller parameters are calculated using following equations: 
$q_{o}=K_{P} \frac{1+2\left(c_{f}+d_{d}\right)+\frac{c_{i}}{2}\left(1+2 c_{f}\right)}{1+2 c_{f}}$

$q_{1}=K_{P} \frac{\frac{c_{i}}{2}-4\left(c_{f}+c_{d}\right)}{1+2 c_{f}}$

$q_{2}=K_{P} \frac{c_{f}\left(2-c_{i}\right)+2 c_{d}+\frac{c_{i}}{2}-1}{1+2 c_{f}}$

$p_{I}=\frac{-4 c_{f}}{1+2 c_{f}}$

$p_{2}=\frac{2 c_{f}-1}{1+2 c_{f}}$

$c_{f}=\frac{T_{f}}{T_{0}}$

$c_{i}=\frac{T_{0}}{T_{i}}$

$c_{d}=\frac{T_{D}}{T_{0}}$

$K_{P}=0.6 K_{P u}$

$T_{I}=0.5 T_{u}$

$T_{D}=0.125 T_{u}$

$T_{f}=\frac{T_{D}}{\alpha}$

where $K_{P}, T_{D}$ and $T_{I}$ are controllers parameters. In (18), $\alpha$ is the filtration coefficient used by filter of process output signal.

\subsection{Online identification methods}

Two methods of identification were used for our simulations purposes, recursive least square method (LSM) and LSM with exponential forgetting. These methods can be used for the discrete on-line identification of processes that are described by the following transfer function $G(z)$ :

$$
G(z)=\frac{B\left(z^{-1}\right)}{A\left(z^{-1}\right)}=\frac{b_{1} z^{-1}+b_{2} z^{-2}+\ldots+b_{m} z^{-m}}{1+a_{1} z^{-1}+a_{2} z^{-2}+\ldots+a_{n} z^{-n}} z^{-d}
$$


The estimated output of the process in the step $k\left(\hat{y}_{k}\right)$ is computed on the basis of the previous process inputs $u$ and outputs $y$ according to the (20):

$$
\hat{y}_{k}=-\hat{a}_{1} y_{k-1}-\ldots-\hat{a}_{n} y_{k-n}+\hat{b}_{1} u_{k-d-1}+\ldots+\hat{b}_{m} u_{k-d-m}
$$

where $\hat{a}_{1}, \ldots \hat{a}_{n}, \hat{b}_{1}, \ldots, \hat{b}_{m}$ are the current estimations of the process parameters. This equation can be also written in vector form, which is more suitable for further work:

$$
\begin{aligned}
& \hat{y}_{k}=\Theta_{k-1}^{T} \cdot \Phi_{k} \\
& \Theta_{k-1}=\left[\hat{a}_{1}, \ldots \hat{a}_{n}, \hat{b}_{1}, \ldots \hat{b}_{m}\right]^{T} \\
& \Phi_{k}=\left[-y_{k-1}, \ldots-y_{k-n}, u_{k-d-1}, \ldots u_{k-d-m}\right] T
\end{aligned}
$$

The vector $\Theta_{k-1}$ contains the process parameter estimations computed in the previous step and the vector $\Phi_{k}$ includes output and input values for computation of current output $y_{k}$.

\subsection{Recursive least square method}

Least square methods are based on minimisation of the sum of prediction errors squares:

$$
J_{k}=\sum_{i=1}^{k}\left(y_{i}-\Theta_{k}^{T} \Phi_{i}\right)^{2}
$$

where $y_{i}$ is the process output in i-th step and the product $\Theta_{k}^{T} \Phi_{i}$ represents the predicted process output. Solving this equation leads to the recursive version of least square method where vector of parameters estimations is updated in each step according to formula:

$$
\Theta_{k}=\Theta_{k-1}+\frac{C_{k-1} \cdot \Phi_{k}}{1+\Phi_{k}^{T} \cdot C_{k-1} \cdot \Phi_{k}} \cdot\left(y_{k}-\Phi_{k-1}^{T} \Phi_{k}\right)
$$

The covariance matrix $C$ is then updated in each step as defined by the equation:

$$
C_{k}=C_{k-1}+\frac{C_{k-1} \cdot \Phi_{k} \cdot \Phi_{k}^{T} \cdot C_{k-1}}{1+\Phi_{k}^{T} \cdot C_{k-1} \cdot \Phi_{k}}
$$

Initial value of matrix $C$ determines the influence of initial parameters estimations on the process identification.

\subsection{Least square method with exponential forgetting}

The main disadvantage of this pure recursive least square method is an absence of signal weighting. Each input and output affect the result by the same weight, but actual process parameters can change in time. Thus newer inputs and outputs should affect the output more than older values. This problem can be solved by exponential forgetting method, which uses forgetting coefficient $\varphi$ and decreases the weights of the data in the previous steps. Weights $\varphi_{k-1}$ are assigned to values $u_{i}$ and $y_{i}$. Parameter estimations are computed according to the following relations:

$$
\begin{aligned}
& \Theta_{k}=\Theta_{k-1}+\frac{C_{k-1} \cdot \Phi_{k}}{\varphi+\Phi_{k}^{T} \cdot C_{k-1} \cdot \Phi_{k}} \cdot\left(y_{k}-\Phi_{k-1}^{T} \Phi_{k}\right) \\
& C_{k}=\frac{1}{\varphi}\left(C_{k-1} \frac{C_{k-1} \cdot \Phi_{k} \cdot \Phi_{k}^{T} \cdot C_{k-1}}{\varphi+\Phi_{k}^{T} \cdot C_{k-1} \cdot \Phi_{k}}\right)
\end{aligned}
$$




\section{Results section}

\subsection{Parameter $\alpha$ adjusting using recursive LSM identification}

First, $\alpha$ filtration coefficient of ZNFD controller was adjusted. In this aspect it must be said that Chalupa [10] introduced the recommended values of parameter $\alpha$ in the interval $(3<\alpha<20)$; however, these values were not suitable in our case. It was necessary to use a far smaller value. In Fig. 2, several temperature profiles with different $\alpha$ filtration coefficients are plotted. As can be seen, the performance of the ZNFD controller is the best for parameter $\alpha=0.003$. In cases of higher $\alpha$, the set point is overshot.

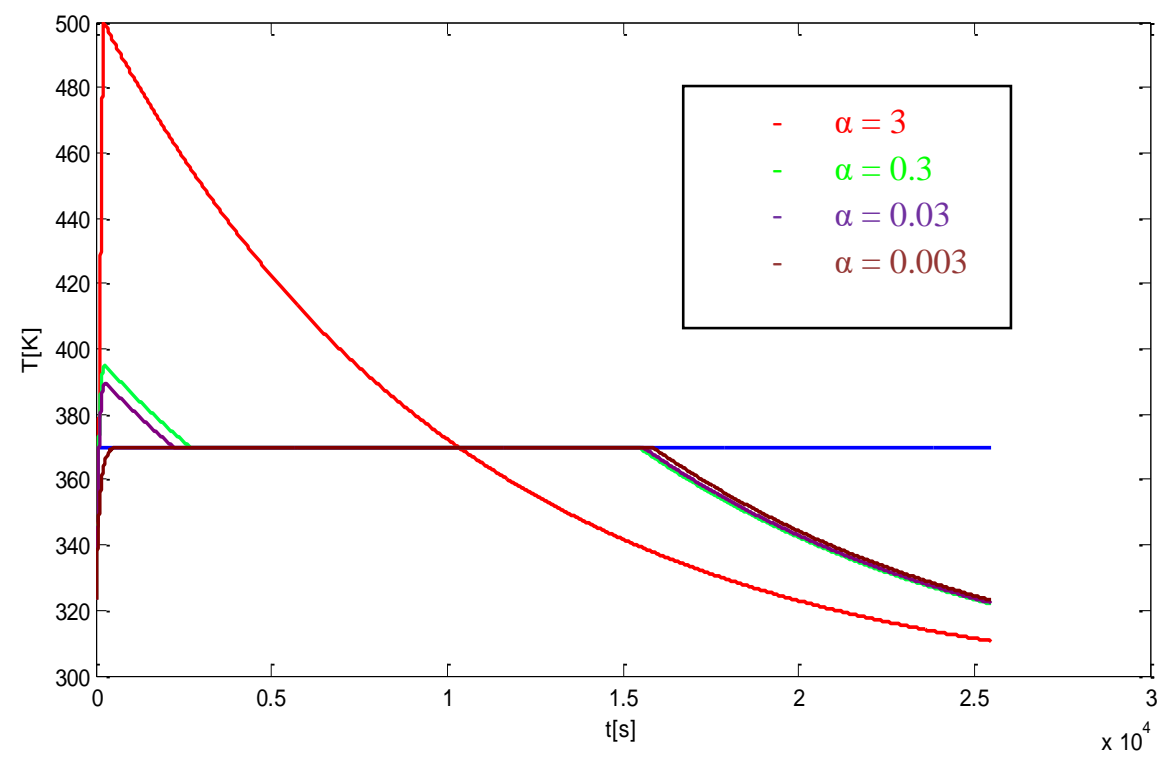

Fig. 2. Comparison of the temperature profiles for different $\alpha$ coefficients

\subsection{Identification using LSM with exponential forgetting}

In the previous section, the controller contains an online identification method - Least squares method (LSM). Here the identification method was extended by exponential forgetting. In order to assess a differences, the parameter $\varphi$ influencing weights of the data from the previous steps, was changed. As can be seen in Fig. 3, the setpoint is reached very quickly without overshoot when $\varphi=1$. It can be noticed that exponential forgetting is not applied when $\varphi=1$, see $(23)-(26)$.

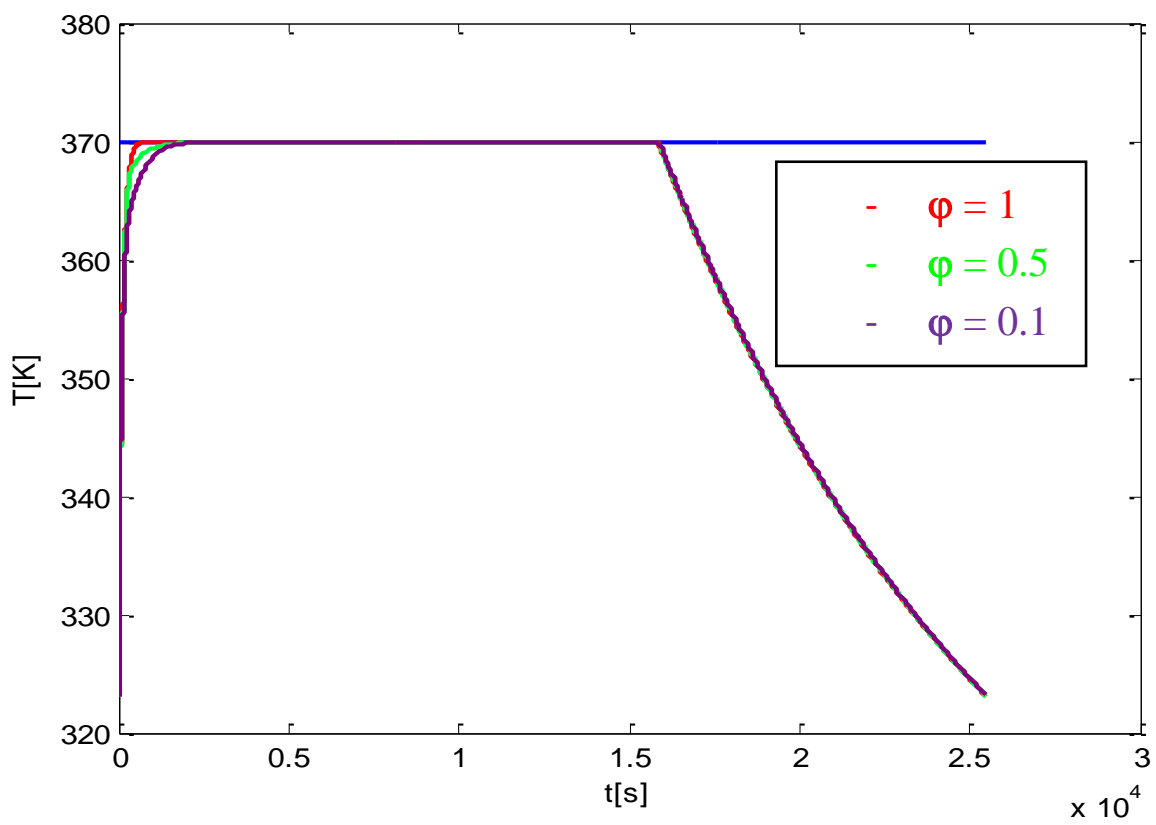

Fig. 3. Comparison of the temperature profiles for different $\varphi$ coefficients 


\subsection{Feeding profiles comparing}

Figure 4 shows that the feeding profile with online identification without exponential forgetting $(\varphi=1)$ is without oscillations. It can be observed that decreasing weights of the data in the previous steps did not improve the temperature and the feeding flow rate profiles in our semi-batch reactor simulations.

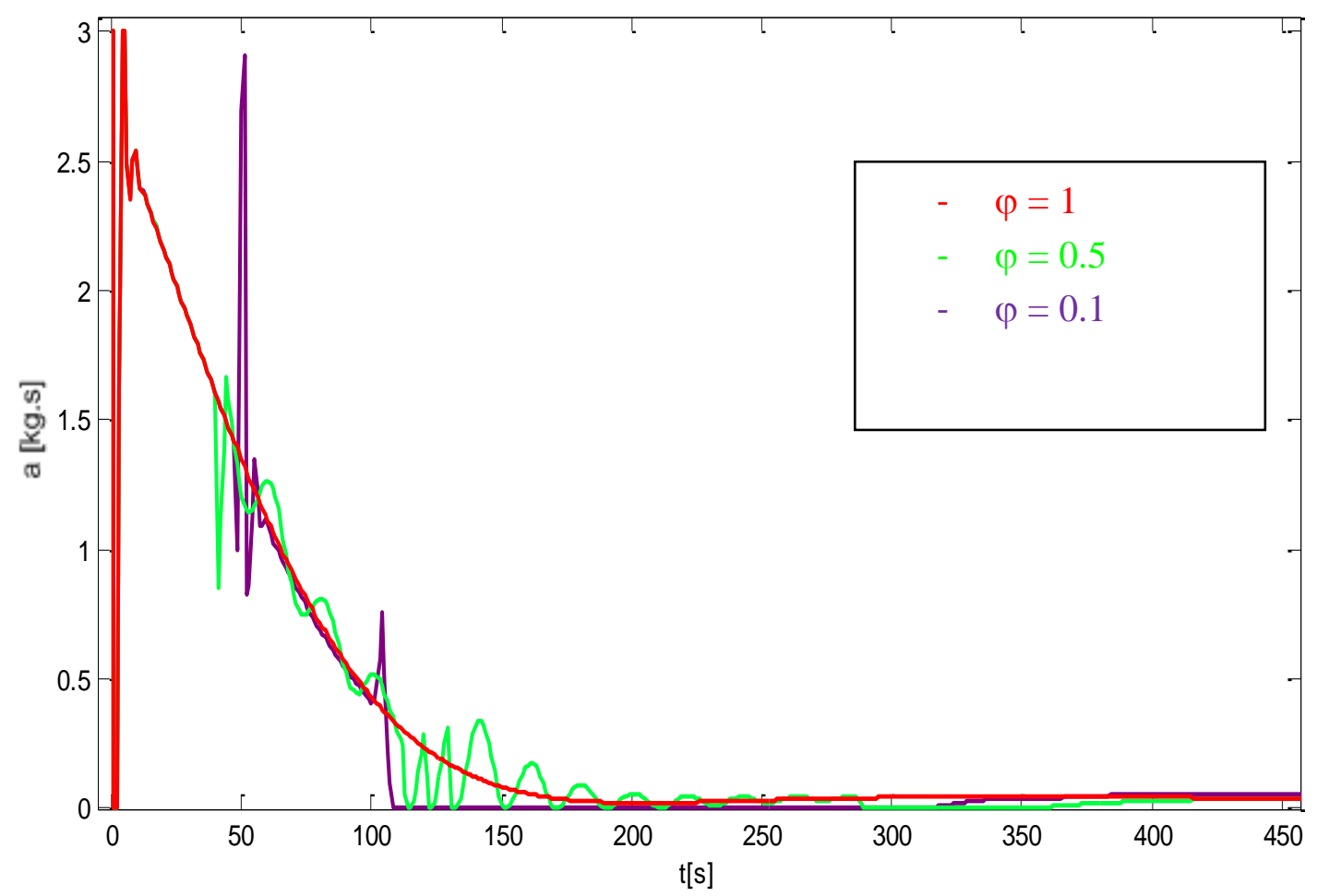

Fig. 4. Feeding profiles comparison for different $\varphi$ coefficients

\section{Conclusion}

Usually some advanced control methods (such a model predictive control) are used for complex systems. These methods can be inevitable for example in case of systems with strong nonlinearities. But, as was shown in this article, sometimes even simple control method can deal with nonlinear systems. Here, the Ziegler-Nichols controller with filtration of D-component using online identification was able to control the semi batch exothermic process. It was visible that this control was closely dependent on the quality of the system identification. Two methods of identification were tested, recursive least square method and least square method with exponential forgetting. However, there was not any visible improvement using the second method, i.e. using the on-line identification with exponential forgetting. It may be caused by the lost ("forgotten") data from the beginning of the process. All previous steps input and output values were needed for good control performance in our semi-batch reactor system.

Future work should focus on the other identification methods verification as well as on the application of these methods to systems with different behaviour, for example flow reactors and the like. Also some general dependences and rules for application these methods to different systems could be observed (if there any).

\section{References}

[1] Zavacka, J. ; Bakosova, M.; Matejickova, K. (2012). Robust PID Controller Design for Unstable Processes with Parametric Uncertainty, Procedia Engineering, Volume 42, p.p. 1572-1578, ISSN 1877-7058, Available from: https://doi.org/10.1016/j.proeng.2012.07.550

[2] Vojtesek, J. (2010). Adaptive Control of Conductivity Inside CSTR, Annals of DAAAM for 2010 \& Proceedings of the 21st International DAAAM Symposium, 20-23rd October 2010, Zadar, Croatia, ISSN 1726-9679, ISBN 978-3901509-73-5, Katalinic, B. (Ed.), pp. 0207-0208, Published by DAAAM International Vienna, Vienna

[3] Plsek, S.; Matusu, R. (2015). Application of Adaptive Dead-beat Controller in Drying Process, Procedia Engineering, Volume 100, p.p. 756-764, ISSN 1877-7058, Available from: https://doi.org/10.1016/j.proeng.2015.01.429 
[4] Bück, A.; Casciatori, F.P.; Thoméo, J.C.; Tsotsas, E. (2015). Model-based Control of Enzyme Yield in Solid-state Fermentation, Procedia Engineering, Volume 102, p.p. 362-371, ISSN 1877-7058, Available from: https://doi.org/10.1016/j.proeng.2015.01.163

[5] Bouhenchir, H.; Cabassud, M.; Le Lann, M.V. (2006). Predictive functional control for the temperature control of a chemical batch reactor, Computers and Chemical Engineering, Issue 30, p.p. 1141-1154

[6] Beyer, M.A.; Grote, W.; Reinig, G. (2008). Adaptive exact linearization control of batch polymerization reactors using a Sigma-Point Kalman Filter, Journal of Process Control, Issue 18, p.p. 663-675, ISSN 0959-1524

[7] Cho, W.; Edgar, T.F.; Lee, J. (2008). Iterative learning dual-mode control of exothermic batch reactors, Control Engineering Practice, 16, p.p. 1244-1249, ISSN 0967-0661

[8] Skrnajc, I. (2007). Self-adaptive supervisory predictive functional control of a hybrid semi-batch reactor with constraints, University of Ljubljana, Slovenia 2007

[9] Wu, X.; Zhang, J.; Zhu, Q. (2010). A generalized procedure in designing recurrent neural network identification and control of time-varying-delayed nonlinear dynamic systems, Neurocomputing, Issue 73, p.p. 1376-1383. ISSN 0925-2312

[10] Bobál, V., Chalupa, P., \& Dostál, P. (2008). Library for design and simulation verification of self-tuning controllers, Proceedings of the 22nd European Conference on Modelling and Simulation (pp. 3-6) 